\title{
Laser-Synthesized SERS Substrates as Sensors toward Therapeutic Drug Monitoring
}

\author{
Matteo Tommasini ${ }^{1}\left(\mathbb{0}\right.$, Chiara Zanchi $^{1,2} \oplus{ }^{10}$, Andrea Lucotti ${ }^{1}\left(\right.$, Alessandro Bombelli ${ }^{2}$,

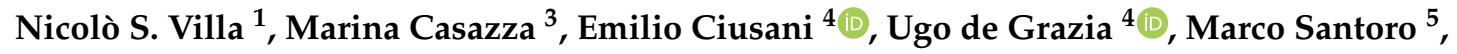 \\ Enza Fazio ${ }^{5}\left(\mathbb{D}\right.$, Fortunato Neri ${ }^{5}\left(\mathbb{D}\right.$, Sebastiano Trusso ${ }^{6}(\mathbb{D})$ and Paolo M. Ossi ${ }^{2, *(D)}$ \\ 1 Dipartimento di Chimica, Materiali e Ingegneria Chimica “G. Natta”, Politecnico di Milano, \\ Piazza Leonardo da Vinci 32, 20133 Milano, Italy; matteo.tommasini@polimi.it (M.T.); \\ chiaragiuseppina.zanchi@polimi.it (C.Z.); andrea.lucotti@polimi.it (A.L.); \\ nicolosimone.villa@mail.polimi.it (N.S.V.) \\ 2 Dipartimento di Energia, Politecnico di Milano, Via Ponzio 34/3, 20133 Milano, Italy; \\ alessandro.bombelli@mail.polimi.it \\ 3 Division of Neurophysiopathology, Fondazione IRCCS Istituto Neurologico Carlo Besta, Via Celoria 11, \\ 20133 Milano, Italy; marina.casazza@istituto-besta.it \\ 4 Laboratorio di Patologia Clinica e Genetica Medica, Fondazione IRCCS Istituto Neurologico Carlo Besta, \\ Via Celoria 11, 20133 Milano, Italy; emilio.ciusani@istituto-besta.it (E.C.); \\ ugo.degrazia@istituto-besta.it (U.d.G.) \\ 5 Dipartimento di Scienze Matematiche e Informatiche, Scienze Fisiche e Scienze della Terra, \\ Università di Messina, Viale Ferdinando Stagno d'Alcontres 31, 98166 Messina, Italy; \\ masantoro@unime.it (M.S.); enfazio@unime.it (E.F.); fneri@unime.it (F.N.) \\ 6 CNR-IPCF, Istituto per i Processi Chimico-Fisici del CNR, V.le F. S. D'Alcontres 37, 98158 Messina, Italy; \\ trusso@ipcf.cnr.it \\ * Correspondence: paolo.ossi@polimi.it; Tel.: +39-02-2399-6319
}

Received: 12 March 2019; Accepted: 19 April 2019; Published: 1 May 2019 updates

\begin{abstract}
The synthesis by pulsed laser ablation and the characterization of both the surface nanostructure and the optical properties of noble metal nanoparticle-based substrates used in Surface Enhanced Raman Spectroscopy are discussed with reference to application in the detection of anti-epileptic drugs. Results on two representative drugs, namely Carbamazepine and Perampanel, are critically addressed.
\end{abstract}

Keywords: noble metal nanoparticles; pulsed laser ablation; surface enhanced Raman spectroscopy; antiepileptic drugs

\section{Introduction}

Research in the field of nanomaterials opens scenarios that are anything but obvious. A particular spectroscopic technique, which aims at identifying and quantifying molecular species of biomedical interest becomes feasible when materials with specifically designed optical properties are available. In principle, it is possible to recognize a chemical species by means of the inelastic scattering of a probing laser light that discloses the features of the vibrational spectrum of the target molecule to be probed. The intrinsic limit of Raman spectroscopy is the very low scattering cross-section (about $10^{-30} \mathrm{~cm}^{2}$ molecule ${ }^{-1}$ ). This may be overcome when a surface plasmon resonance (SPR) and the associated strong increase of the scattered intensity is triggered at a nanostructured metal surface (Surface Enhanced Raman Spectroscopy, SERS). The wavelength and Full Width at Half Maximum (FWHM) of the SP, as recorded in the UV-Vis spectrum of the film, are the optical characteristics of a corrugated metal surface of specific interest when exploring its behavior as an active SERS substrate. 
Near the metal surface, the electric field enhancement associated with the exciting light is most relevant with the noble metals silver and gold. In particular, large electromagnetic field enhancements [1,2] are observed at hot spots, corresponding to specific local surface morphologies that include sharp tips, edges and thin interparticle gaps. The surface nanostructures of artificially roughened Ag and $\mathrm{Au}$ thin films display many hot spots [3] making them excellent SERS substrates. Thus, the goal is to synthesize Ag and Au films with surface nanostructure engineered so as to maximize the SERS signal.

Pulsed laser ablation (PLA) makes use of two alternative methods to produce artificially corrugated nanostructured surfaces. Both methods are based on the vaporization of a solid target by high-energy laser pulses in an ambient fluid, whose role is to confine the vaporized species and to induce their mutual aggregation, obtaining clusters and nanoparticles (NPs) without chemical precursors. The fluid can be a gas or a liquid transparent to the laser radiation.

When ablation is performed using an ns-laser (nanosecond-laser) in a high pressure, neutral ambient gas, NPs are directly deposited onto appropriate inert supports where they self-arrange leading to qualitatively different surface nanostructures [4-6]. For defined experimental conditions [4], a path connects two extreme morphologies: isolated, sphere-like NPs, more or less crowded together, and a continuous metal film. The two relevant parameters to design the surface nanostructure of the growing film are the number of laser pulses $\left(N_{L P}\right)$ and the ambient gas pressure $\left(p_{g}\right)$. In fact, for a progressively increasing $N_{L P}$ and $p_{g}$, we observe first particles more and more coalesced together whose size increases progressively while the shape departs from spherical and becomes more and more irregular. Later, islands separated by a network of randomly oriented channels with variable length and width in the few nm range develop. By increasing $p_{g}$, keeping constant $N_{L P}$ at low values, the support is covered by increasingly smaller, spherical NPs; at high $N_{L P}$ values, we observe islands with smaller average size and correspondingly a larger number of shorter channels (see Figure 1a,b). The $N_{L P}$ value defines the degree of support coverage. With increasing $N_{L P}$, keeping constant $p_{g}$, we observe a spatial densification of the NPs on the support. The role of $N_{L P}$ and $p_{g}$ is illustrated in Figure 1 for self-assembled Au NPs on (100) Si supports. The same qualitative trend is observed for Ag NPs.

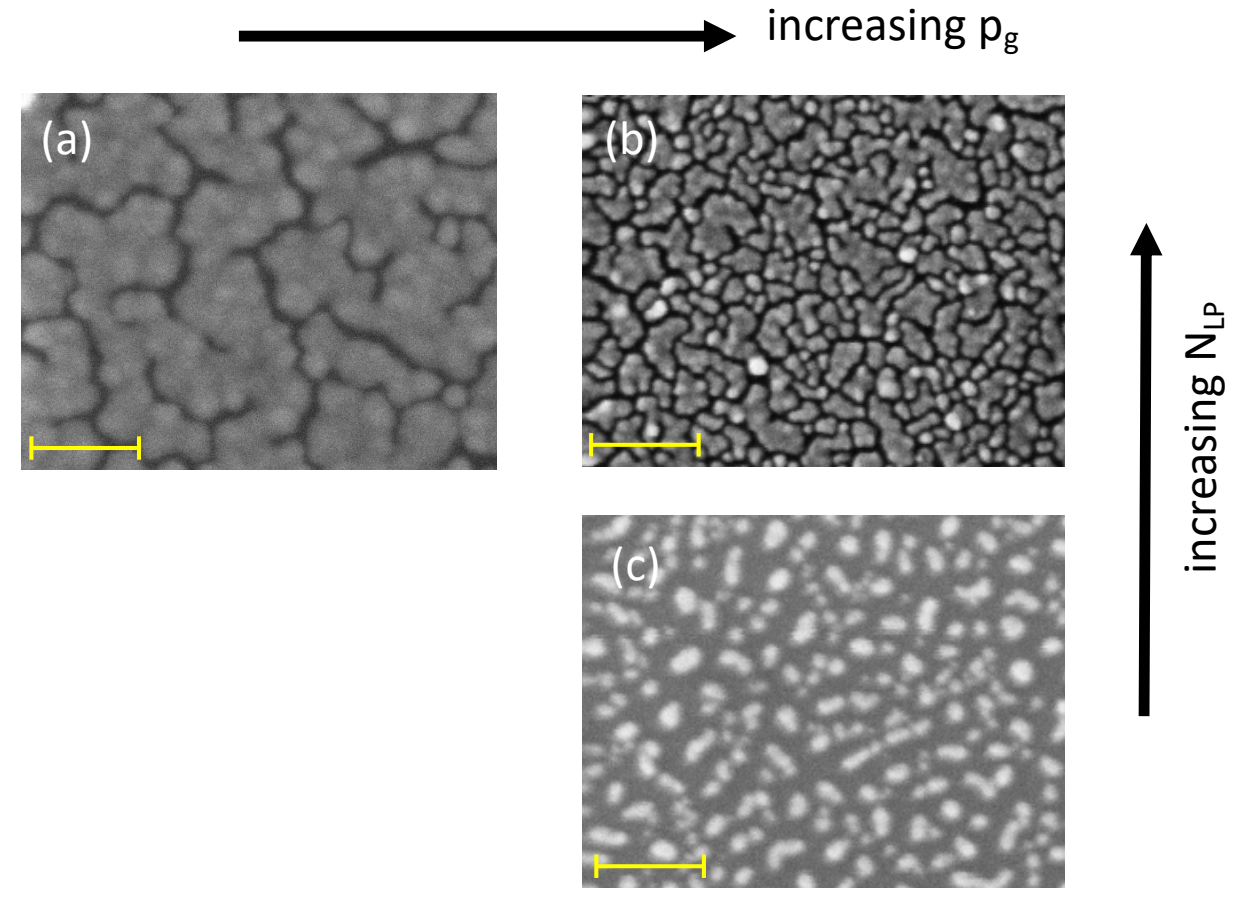

Figure 1. Scanning Electron Microscope (SEM) micrographs showing the dependence of the surface nanostructure of $\mathrm{Au}$ films deposited in $\mathrm{Ar}$ on the ambient gas pressure $\left(p_{g}\right)$ and on the number of laser pulses $\left(N_{L P}\right)$. (a) $N_{L P}=3 \times 10^{4}, p_{g}=10 \mathrm{~Pa}$; (b) $N_{L P}=3 \times 10^{4}, p_{g}=100 \mathrm{~Pa}$; (c) $N_{L P}=5 \times 10^{3}, p_{g}=100$ $\mathrm{Pa}$. The yellow bars correspond to $100 \mathrm{~nm}$. See Experimental for details. 
Remarkably, for both metals, the average NP sizes observed in the Transmission Electron Microscope (TEM) agree with the predictions of a model for the plasma expansion through a high-pressure ambient gas [4,7]. Concerning the optical properties of the obtained nanostructures, for both metals, the SP wavelength decreases with increasing $p_{g}$ at fixed $N_{L P}$, as well as, when $p_{g}$ is kept fixed and $N_{L P}$ is increased, the SP wavelength increases.

When a liquid is used as the confining medium through which the ablation plasma expands, a colloidal solution is obtained. By pulsed laser ablation in liquid (PLAL), using a nano/picosecond laser source, surfactant-free NPs are produced in a single-step approach within a time scale of a few minutes [8]. In a stationary liquid, the process consists of the production of NPs by ablation of the target, and the contemporaneous fragmentation-assembling of dispersed NPs by continuous irradiation of already synthesized particles. The laser pulse duration is a relevant parameter for NP generation [9]. With picosecond pulses (ps pulses), the relevance of melting and thermal evaporation is strongly reduced with respect to nanosecond pulses (ns pulses). With short pulses, the ablation process becomes increasingly efficient involving a nearly instantaneous vaporization with minimized heat-affected zone [10], so that the colloidal solution is produced in a shorter time. Besides this, compared to ns pulses, ps pulses allow for mitigating primary plasma shielding that is detrimental to ablation efficiency [11].

Recently, in the scientific literature that deals with drug dosage [12], there emerged a promising technique based on Raman/SERS, specifically suited to low drug concentrations [13-17] that is complementary to those in clinical use. In a potential application scenario, it would be possible to trace the concentration of a drug in blood plasma samples of clinical origin subjected to limited and rapid treatments (e.g., centrifugation, extraction with solvents). The SERS measurement exploits the interaction of the drug molecule with a nanostructured metal surface and the intensity of the signal, under controlled conditions, allows for tracing the quantity of molecules adsorbed on the metal, which is a function of the drug concentration in the tested solution. The analytical capability of SERS was shown to be comparable with High-Performance Liquid Chromatography (HPLC) technique [18], thus making possible to study the potentiality of this spectroscopic technique in the field of Therapeutic Drug Monitoring (TDM). TDM is a clinical practice that involves determining the concentration of a drug in a biological fluid, usually blood plasma. This procedure is particularly relevant for drugs characterized by a narrow therapeutic index (NTI). In such drugs, the difference between the concentration at which therapeutic effects and the (slightly larger) concentration at which side effects are observed for the patient is minimal. In therapies that use NTI drugs, it is important to know the plasma concentration of the drug. This way, the patient's clinical conditions can be associated with the required drug dose to guarantee the effectiveness of the treatment, avoiding the occurrence of side effects. TDM has been used for a long time in the clinical practice for NTI drugs. Among these drugs, we find anticancer drugs and antiepileptic drugs (AEDs). For the latter, toxicity can be induced by a small drug excess, whereas even a small reduction of the effective dosage can reduce the efficiency in controlling seizures.

Current research efforts in the SERS-TDM field point at overcoming the difficulties associated with the weakness of the signals of some drugs and/or to the background signal from much more abundant biomolecules coexisting with the drug in the fluid to be analyzed. A second relevant research direction is the development of SERS sensors (i.e., nanostructured metal surfaces) that combine a high sensitivity with spatial uniformity, control and reproducibility of the manufacturing process, not disregarding a low-cost production. Before the SERS technique can be routinely introduced into the clinical laboratory, besides the above technological issues, the treatment of the samples should also be optimized. Finally, validation procedures are required to develop quantitative SERS measurements. These imply determining drug concentration with standard reference methods (HPLC-MS or immunological assay).

In this work, we show how noble metal thin films resulting from self-assembled NPs can be produced in a controlled and reproducible way by PLA techniques. The obtained surface nanostructures are irregular and non-periodic. The peculiar morphological features of such 
nanostructures allow the good stability and reproducibility of the SPR of the films and lead to optimal electromagnetic enhancements in SERS [2]. Such control on plasmonic properties is required for effective application of SERS in analytics [14,19]. We have employed these films in TDM, focusing on two AEDs (Carbamazepine-CBZ, Perampanel—PER). In the case of CBZ, we prove that a SERS substrate can be re-utilized at least five times by washing it with methanol. We also show the good linear dependence of the SERS signal vs. CBZ concentration in the range $2.5 \times 10^{-5} \mathrm{M}$ to $2.1 \times 10^{-4} \mathrm{M}$. In the case of PER, we exploit the protonation mechanism of the drug by $\mathrm{HCl}$, as suggested by observed changes of the $\mathrm{C}=\mathrm{O}$ stretching transition between Raman and SERS, and UV-Vis data taken on PER in acidic conditions. This paves the way to the control of the chemical enhancement pathway in SERS, which is triggered by effective chemical interaction of the analyte and the noble metal surface.

\section{Experimental}

\subsection{Production of Au Substrates by PLA in High-Pressure Inert Gas}

$\mathrm{Au}$ films were prepared at room temperature in a vacuum chamber, starting from a base pressure lower than $10^{-4} \mathrm{~Pa}$ using a $\mathrm{KrF}$ excimer laser (wavelength $248 \mathrm{~nm}$, pulse width $25 \mathrm{~ns}$, repetition rate $10 \mathrm{~Hz}$, incidence angle $45^{\circ}$ ) focused onto an elemental target ( $\left.\mathrm{Au}, 99.99 \%\right)$ mounted on a rotating holder. The films were deposited onto pieces of glass, or (100) Si placed in front of the target at a distance of $35 \mathrm{~mm}$. The target holder was rotated to avoid cratering of the target surface under repetitive ablation. Ablation was performed in Ar atmosphere at $100 \mathrm{~Pa}$, with $N_{L P}$ fixed at $2 \times 10^{4}$, and the laser fluence $(f)$ kept constant at $f=2.0 \mathrm{~J} \cdot \mathrm{cm}^{-2}$. Sample surface nanostructuring was observed by scanning electron microscopy (SEM) using a Zeiss Supra 40 field ion microscope (Carl Zeiss NV, Via Varesina 162, 20156 Milano, Italy). UV-vis spectroscopy measurements of SPR were performed with a PerkinElmer UV-Vis/NIR Lambda 750 spectrophotometer (PerkinElmer Italia Spa, Viale dell'Innovazione 3, 20126 Milano, Italy) over the range $190-900 \mathrm{~nm}$.

\subsection{Production of Ag Colloids by PLAL using Water}

We carried out PLAL of an elemental target $(\mathrm{Ag}, 99.9 \%)$ in deionized water using the second harmonic $(532 \mathrm{~nm})$ of a laser operating at $100 \mathrm{kHz}$ repetition rate with pulse width of 6-8 ps. Ablation was performed at $f=1.5 \mathrm{~J} \cdot \mathrm{cm}^{-2}$, for an irradiation time of $10 \mathrm{~min}$. The laser beam was focused with a galvanometric scanner to a spot of about $80 \mu \mathrm{m}$ in diameter on the surface of the target that was scanned on a $10 \times 10 \mathrm{~mm}^{2}$ area with a scan speed of $800 \mathrm{~mm} \cdot \mathrm{s}^{-1}$. The colloids were transferred on glass, or (100) Si supports by an ultrasonic spray-casting deposition method. The experimental setup consists of a deposition chamber equipped with an ultrasonic atomizer (Sonics VCX $130 \mathrm{~W}$, Sonics \& Materials Inc., 53 Church Hill Rd, Newtown, CT 06470, USA), a heated substrate holder and a system to remove excess vapors, thus guaranteeing standard and reproducible conditions. By ultrasonic spraying, we deposited a fraction of the produced colloids on nickel grids to perform Scanning Transmission Electron Microscopy (STEM), using an instrument operating at the primary accelerating voltage of $30 \mathrm{kV}$, at a working distance of $4 \mathrm{~mm}$ (Zeiss model Merlin Gemini 2).

By the same ultrasonic spraying procedure, we deposited Ag NPs onto (100) Si supports, obtaining substrates suitable for SERS measurements. In Figure 2, we show a representative STEM image (a), the average size distribution (b), and the optical absorbance spectrum (c) of Ag colloids prepared by ps-PLAL in water at the optimized laser fluence $f=1.5 \mathrm{~J} \cdot \mathrm{cm}^{-2}$. Nearly spherical NPs, whose size is about $15 \mathrm{~nm}$, result from the likely agglomeration and overlap of smaller NPs (see Figure 2a). The UV-Vis absorption spectrum (Figure 2c) displays a narrow SPR, as expected on the basis of the narrow size distribution of the constituent NPs. This is the outcome of the optimization of the laser fluence. In the range $0.5 \mathrm{~J} \cdot \mathrm{cm}^{-2} \leq f \leq 1.5 \mathrm{~J} \cdot \mathrm{cm}^{-2}$, the SPR peak intensity increases and its FWHM decreases on increasing the laser fluence, keeping fixed all other deposition parameters. 

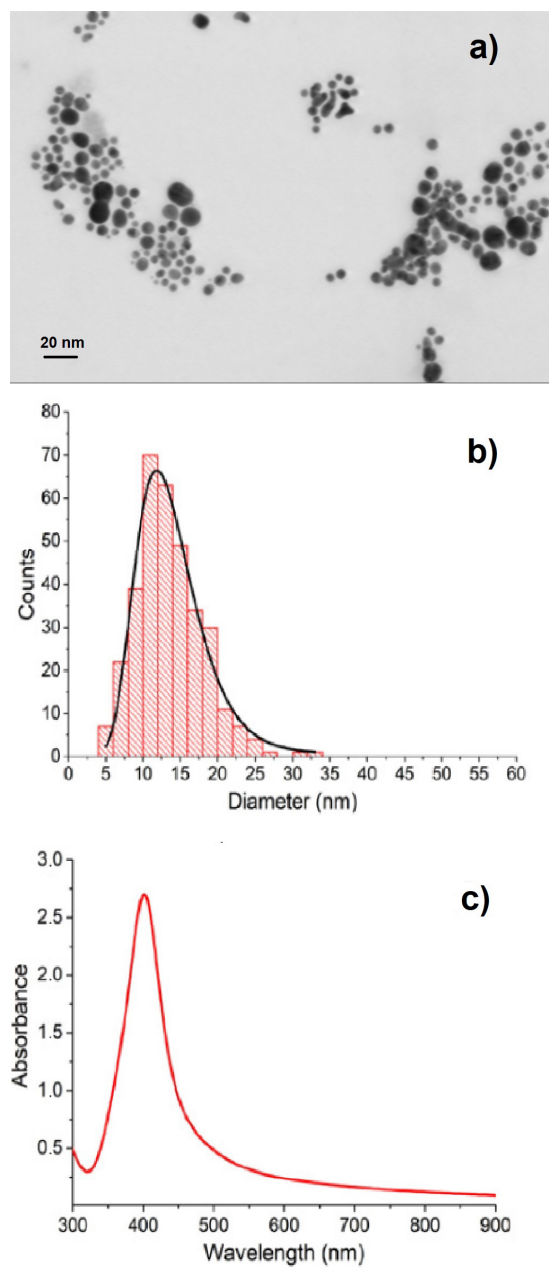

Figure 2. (a) Scanning Transmission Electron Microscope (STEM) images; (b) size distribution of nanoparticles (NPs); (c) optical absorbance spectrum of Ag colloids prepared by picosecond pulsed laser ablation in liquid (ps-PLAL) at the laser fluence of $1.5 \mathrm{~J} \cdot \mathrm{cm}^{-2}$.

\subsection{Raman Spectroscopy}

Raman and SERS spectra were collected by a HORIBA Jobin-Yvon LabRAM HR800 Raman Spectrometer(HORIBA France SAS, 231 rue de Lille, 59650 Villeneuve d'Ascq, France) with a solid-state laser (Laser XTRA, Toptica Photonics, TOPTICA Photonics AG, Lochhamer Schlag 19, 82166 Graefelfing (Munich), Germany) operating at $785 \mathrm{~nm}$, equipped with a 600 grooves $\cdot \mathrm{mm}^{-1}$ grating, a Peltier-cooled Charge-Coupled Device (CCD) detector, and notch filters to suppress Rayleigh scattering contributions. The same Raman spectrometer can be operated also with the $458 \mathrm{~nm}$ excitation from an Ar-ion laser.

\section{Results and Discussion}

The ability to produce nanostructured surfaces with a highly uniform morphology allows for designing sensors with a SERS signal of adequate reproducibility. This condition is mandatory when such sensors are used to detect analytes at low concentrations (as for drugs). In the recent past, we developed two complementary approaches to produce SERS sensors by exploiting the remarkable control in the production of nanoparticles offered by laser ablation techniques. The high control and reproducibility of these manufacturing processes allow the production of nanomaterials with optimized morphology, high sensitivity and spatial uniformity.

The first approach discussed in Section 3.1 below employs ablation in high-pressure inert gas to produce Au sensors, which we tested on the AED Carbamazepine (Figure 3a). In the second approach, 
which we considered at a later time, Ag colloids are produced by ablation of the target in water, and are subsequently sprayed on a support to obtain the thin film SERS sensor. We show in Section 3.2 below our early and promising results obtained by testing these PLAL sensors on a second AED, namely Perampanel (Figure $3 \mathrm{~b}$ ). The choice of $\mathrm{Ag}$ allows for using $\mathrm{HCl}$ to control $\mathrm{pH}$, fostering protonation of the drug, and providing chloride ions which are known to promote SERS action on silver [20]. Carbamazepine (CBZ) is a well-established drug largely used in developing Countries, whereas Perampanel (PER) is a new generation AED.<smiles>NC(=O)N1c2ccccc2C=Cc2ccccc21</smiles>

(a)<smiles>N#Cc1ccccc1-c1cc(-c2ccccn2)cn(-c2ccccc2)c1=O</smiles>

(b)

Figure 3. The chemical structure of (a) Carbamazepine, and (b) Perampanel.

\subsection{Quantitative SERS Detection of Carbamazepine}

Nanostructured films made of arrays of NPs produced by Pulsed Laser Deposition (PLD) of a solid Au target in high-pressure inert gas and mutually assembled on an inert (glass) support behave as SERS sensors with good performances [16].

In Figure 4, we show the surface nanostructure of an Au film deposited on (100) Si (ablation in Ar at $100 \mathrm{~Pa} ; N_{L P}=2 \times 10^{4}$ ). As shown in Figure 4, the SPR of films produced with this set of process parameters and deposited on glass matches the popular $785 \mathrm{~nm}$ laser excitation found in commercial Raman instruments, including portable ones. We used substrates of this kind, either deposited on glass, or on $\mathrm{Si}$, throughout all investigations on CBZ. Part of the data we discuss on CBZ was presented in [6]. Here, we complete the data analysis including additional SERS markers, and we show in detail the evolution of SERS markers upon the washing procedure of a sensor.
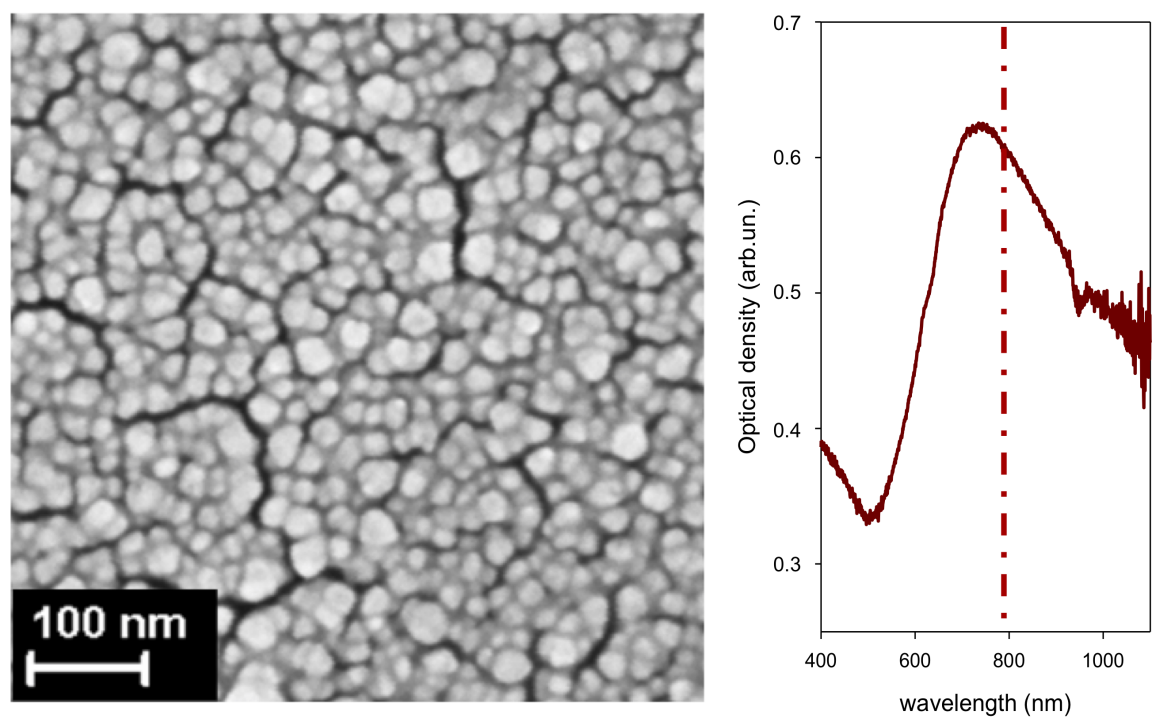

Figure 4. Surface nanostructure of an Au substrate deposited on (100) Si (ablation in Ar at $100 \mathrm{~Pa}$, $\left.N_{L P}=2 \times 10^{4}\right)$. The pertinent UV-Vis spectrum with the position of the exciting laser radiation $(785 \mathrm{~nm})$ is reported. 
As reported elsewhere [16], there is agreement between the Raman and SERS features of CBZ over a wide wavenumber range. This is suggestive of a comparatively weak interaction between the Au substrate and the CBZ molecule. This fact, together with the remarkable stability of the substrates opens the way to recycle them, adopting a washing procedure with $\mathrm{MeOH}$ between consecutive measurements of drug concentration. The results of such washing procedure are shown in Figure 5. These results prove the following: (i) the background of the pristine sensor is blank in the spectral region of the CBZ markers; (ii) washing the sensor by immersion in $\mathrm{MeOH}$ for 5 min effectively removes the drug from the active surface as supported by the disappearance of the SERS markers; (iii) the successive immersion of the sensor in CBZ solution restores the initial SERS signal (i.e., the functioning of the sensor is preserved, even after five cycles of operation-which is enough to obtain the calibration curve of the sensor for four different values of concentration, see below); (iv) the interaction between $\mathrm{CBZ}$ and $\mathrm{Au}$ is weak (physisorption). Point (iv), together with the large wavelength distance between the absorption peak of CBZ (285 nm-see Figure 6) and the plasmon resonance (735 nm, very close to the laser excitation at $785 \mathrm{~nm}$ ), indicate that the SERS of CBZ is electromagnetic in nature [21].
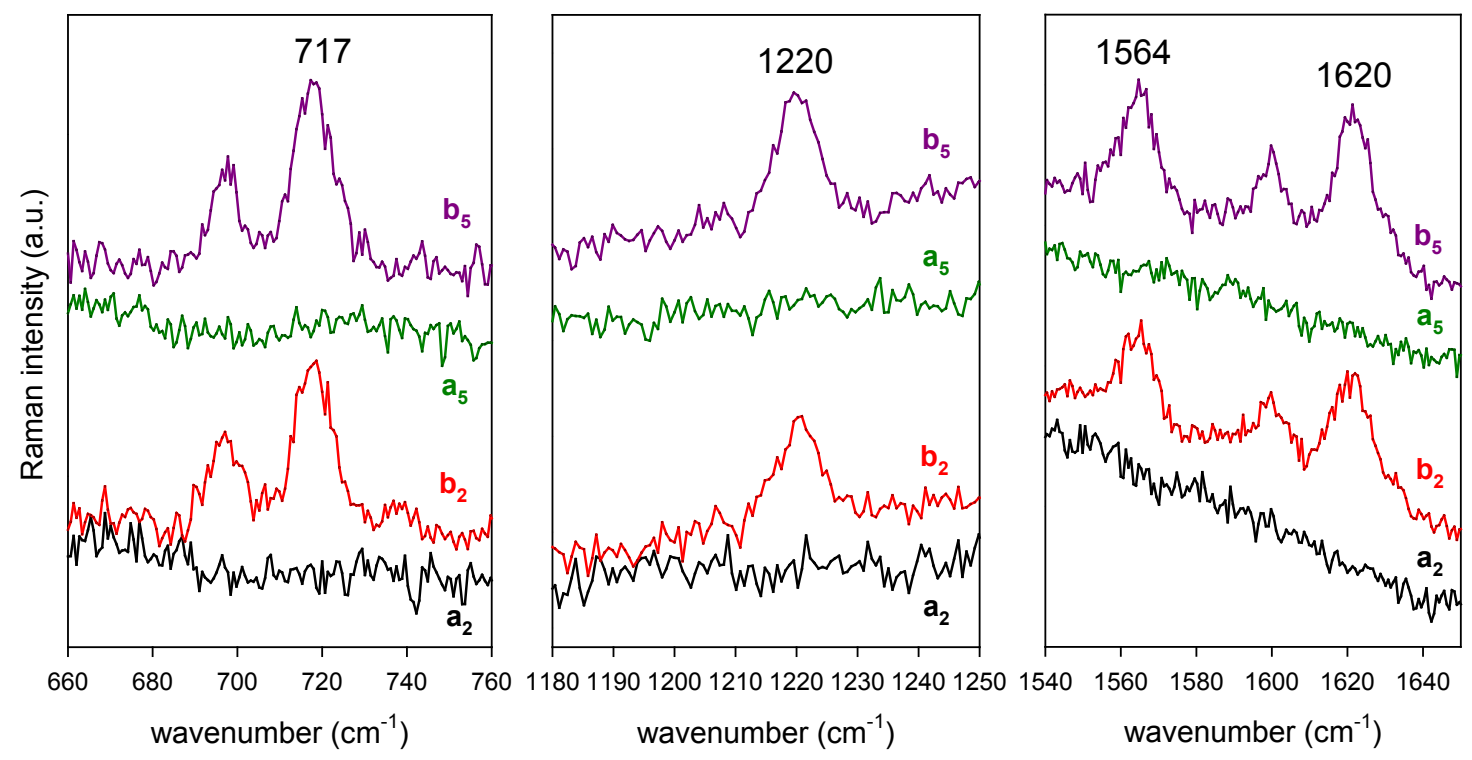

Figure 5. Effect of repeated immersions of a single Au SERS sensor (deposition on glass; ablation in Ar at $\left.100 \mathrm{~Pa}, N_{L P}=2 \times 10^{4}\right)$ in a concentrated CBZ solution (100 mg/L in $\mathrm{MeOH}, 60 \mathrm{~s}$ immersion time) and subsequent washing with $\mathrm{MeOH}$. The labels indicate the spectrum recorded after washing with $\mathrm{MeOH}(\mathbf{a})$ and after immersion in the CBZ solution (b). The pedix is the step number of this repeated sequence of experiments.

After proving the re-usability of such SERS sensors, we quantified their response as a function of CBZ concentration in a range which includes the therapeutic range $\left(2.5 \times 10^{-5} \mathrm{M}-5.1 \times 10^{-5} \mathrm{M}\right)$. Out of the seven available SERS markers [16], we selected those which show up more clearly from the background $\left(717,1220,1564\right.$ and $1619 \mathrm{~cm}^{-1}$ —see Table 1). The measurements shown in Figure 7 were performed by using one single substrate at increasing CBZ concentration. Spectra were recorded by taking 3 averages of $10 \mathrm{~s}$ each. The laser power at the sample surface was $1 \mathrm{~mW}$ over a spot area of $1 \mathrm{um}$ in diameter. The substrate was dipped for $5 \mathrm{~s}$ in a $1 \mathrm{~mL}$ volume of the CBZ solution, and dried before recording. The substrate was washed in $\mathrm{MeOH}$ for $5 \mathrm{~min}$ after each SERS measurement. Control SERS spectra were taken after each washing procedure showing the complete disappearance of all CBZ features. The sensitivity to drug concentration is evident in Figure 7 for all the selected CBZ markers. 
Table 1. Assignment of the SERS markers of Carbamazepine (CBZ) selected in this work (after [16]).

\begin{tabular}{cl}
\hline Wavenumber $\left(\mathbf{c m}^{\mathbf{- 1}}\right)$ & Description \\
\hline 717 & C-H out-of-plane bending at $\mathrm{C}=\mathrm{C}$ and aryl groups \\
1220 & C-H in-plane bending at C=C and aryl groups \\
1564 & ring stretching \\
1619 & C=C stretching \\
\hline
\end{tabular}

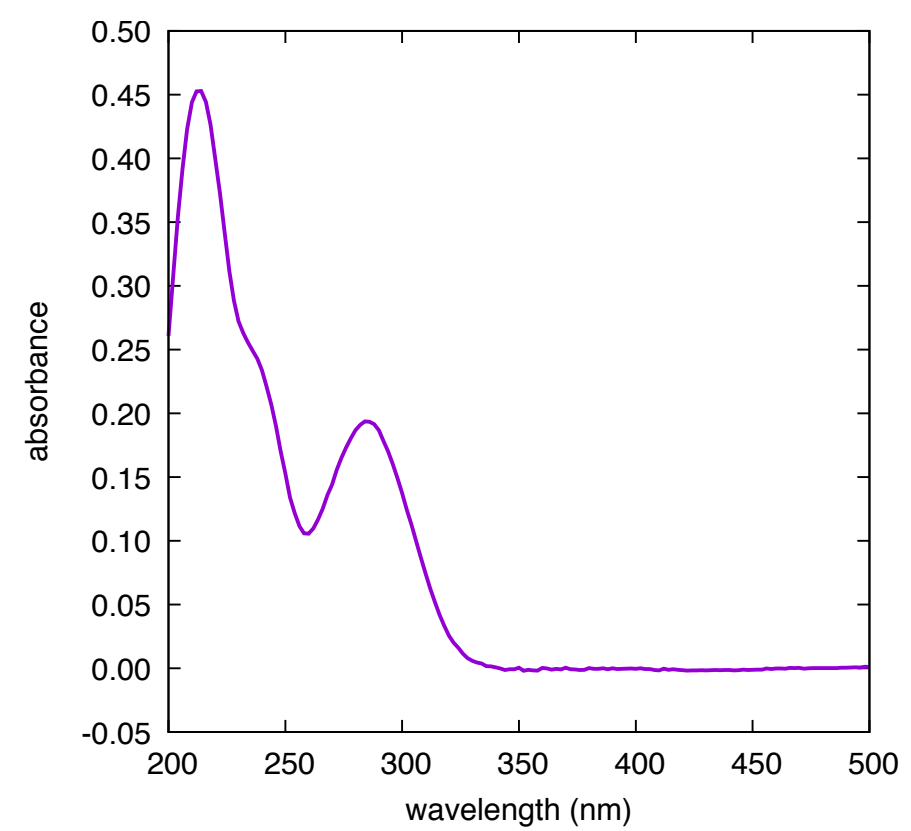

Figure 6. UV-Vis absorption spectra of a methanol solution of Carbamazepine (CBZ) $\left(2.6 \times 10^{-5} \mathrm{M}\right.$, $1 \mathrm{~cm}$ pathlength quartz cuvette, Jasco V-570 spectrometer (Jasco Europe S.R.L., Via Luigi Cadorna 1, 23894 Cremella (LC), Italy)).

In Figure 8, we report the SERS spectrum of CBZ at the total concentration of $5.0 \times 10^{-5} \mathrm{M}$ in blood serum from an epileptic patient. We used an Au substrate deposited on (100)Si to record the SERS spectrum adopting a higher laser power $(10 \mathrm{~mW})$ and a longer integration time (100 s). We observe four SERS signatures coincident with CBZ signatures, notwithstanding the large fraction (about 70\%) of CBZ bound to albumin, as well as the complexity of a biological matrix such as the blood serum has. It is interesting to notice that the peculiar nanostructure of our SERS sensors produced by PLD in gas results in plasmonic hot spots localized at the narrow (few nm wide) channels that separate from each other $\mathrm{Au}$ islands made by agglomerated NPs on the support [2]. When samples are composed by molecules characterized by different molecular weights, and thus different diffusivities, this unique substrate morphology may enhance the probability that small molecules (such as drugs) reach the hot spots with respect to more bulky species. 
(a)
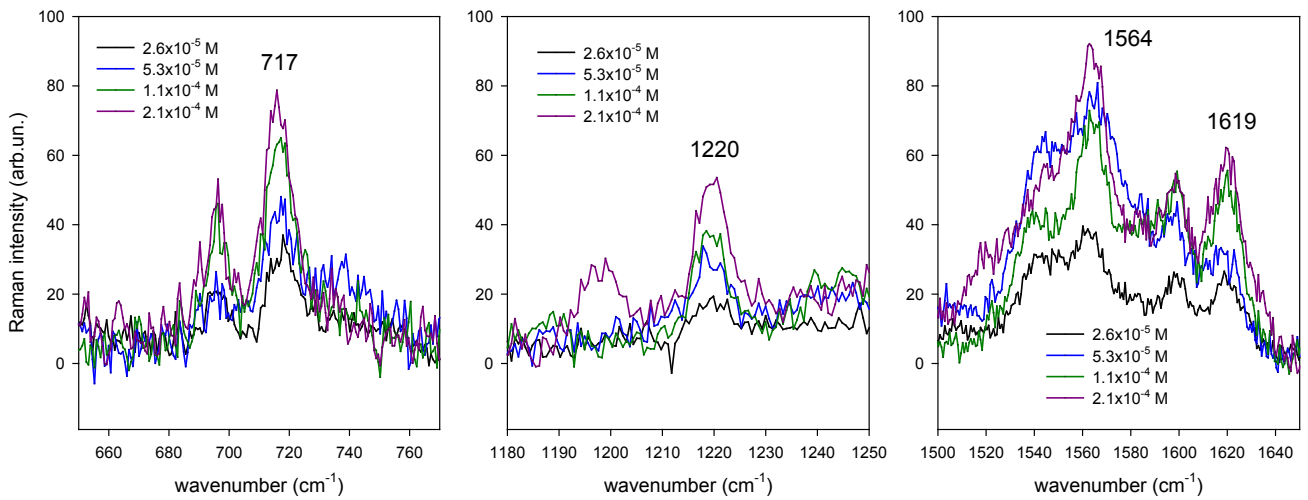

(b)
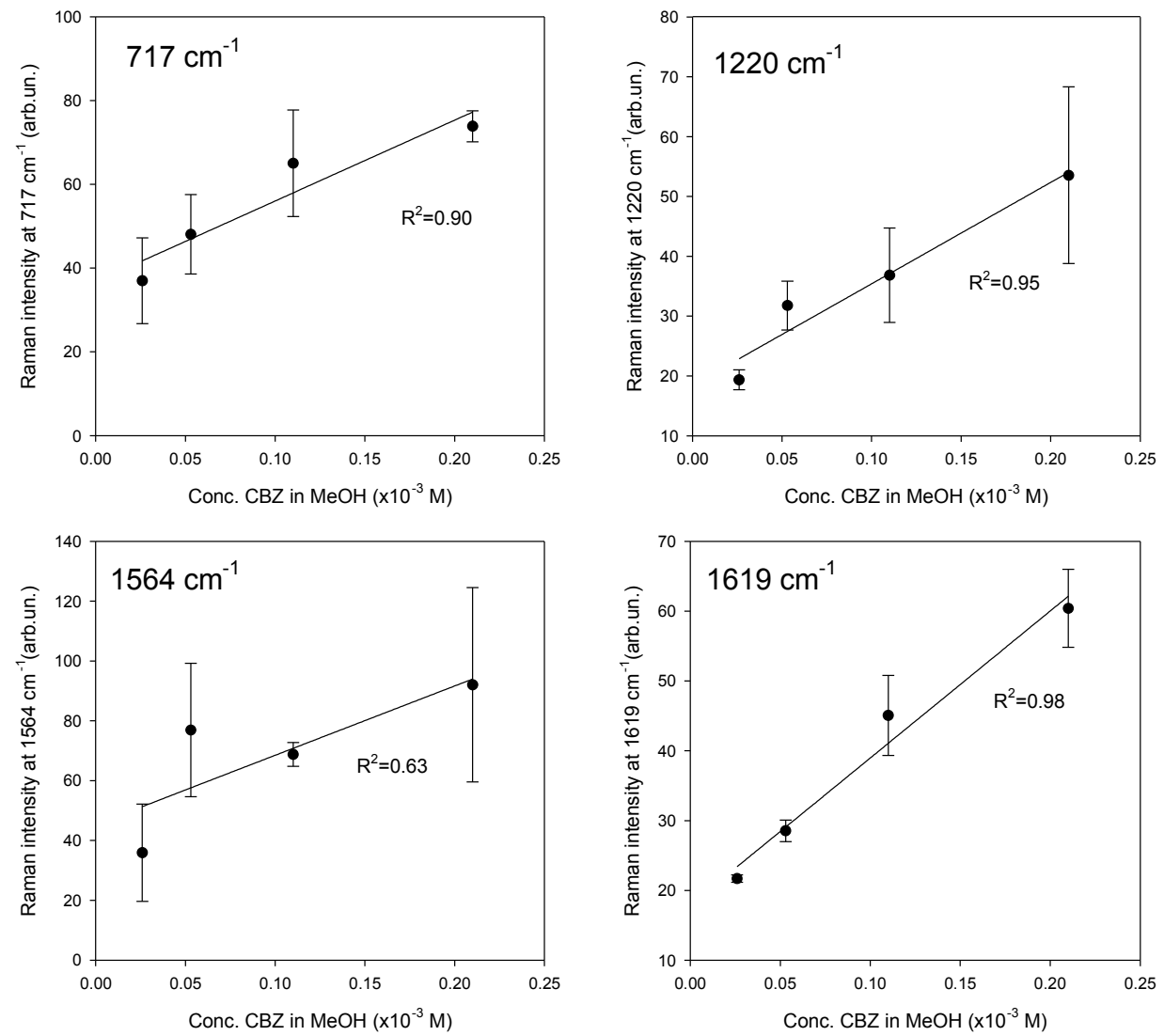

Figure 7. (a) average SERS spectra of $\mathrm{CBZ}$ in $\mathrm{MeOH}$ at different concentrations including the therapeutic range, in selected regions where the four CBZ markers are located $(717,1220,1564$ and $\left.1619 \mathrm{~cm}^{-1}\right)$; (b) calibration curves obtained by averaging the SERS intensities recorded at each peak vs. the corresponding CBZ concentration (range $2.5 \times 10^{-5}$ to $2.1 \times 10^{-4} \mathrm{M}$ ). Averages were done after baseline subtraction over a minimum of 3 to a maximum of 5 spectra, depending on concentration; vertical bars are the corresponding standard deviations. 


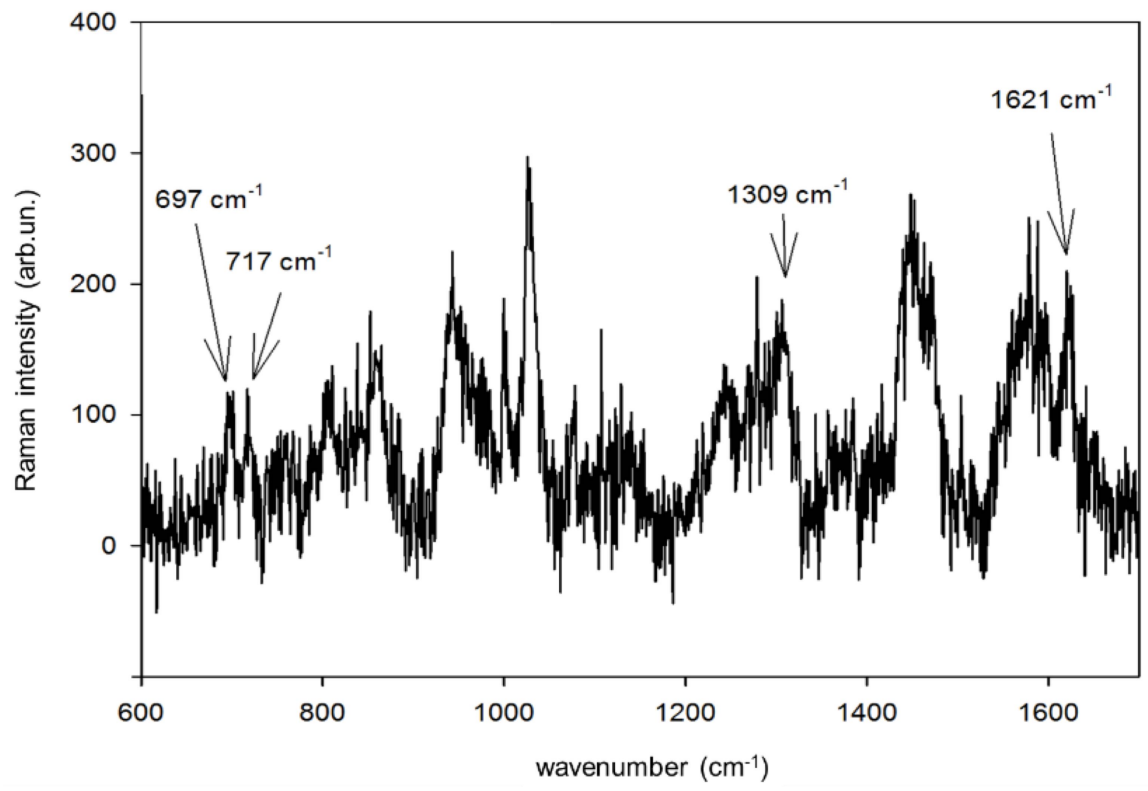

Figure 8. SERS spectrum of CBZ at the total concentration of $5.0 \times 10^{-5} \mathrm{M}$ in blood serum, recorded on an Au substrate deposited on (100)Si (Ar at $\left.100 \mathrm{~Pa}, N_{L P}=2 \times 10^{4}\right)$.

\subsection{SERS of Perampanel in Acidic Water Solutions}

For SERS measurements of PER, we used an Ag substrate ultrasonically sprayed on a (100)Si support starting from colloids synthesized by ps-PLAL (see Experimental). The surface nanostructure (Figure 9a) consists of nearly spherical NPs in part coalesced to give spheroidal shapes and more complex agglomerates. The UV-Vis absorption spectrum from a companion film sprayed on glass is shown in Figure 9b.

Moving from our previous observation that an acidic environment (using $\mathrm{HCl}$ ) fosters SERS on such Ag substrates through the protonation of PER, extracted from Fycompa ${ }^{\circledR}$ tablets [22], we prepared aqueous solutions of PER (Cayman Chemical Item No. 23003; CAS 380917-97-5) at the concentration of $5 \times 10^{-5} \mathrm{M}$ at different $\mathrm{pH}$ values, starting from a concentrated methanol solution of PER in water acidified with $\mathrm{HCl}$. The $\mathrm{pH}$ was checked each time before adding the drug. Our preparation procedure of PER solutions suggests that the charge state of PER plays a relevant role to SERS measurements, thus making evident the importance of the chemical enhancement mechanism [23].

In Figure 10, we show that the absorbance of PER displays a systematic dependence on the $\mathrm{pH}$ of the solution. The protonation process appears to start at $\mathrm{pH} 3$, and is achieved at $\mathrm{pH} 2$ (and lower). We tested the SERS performance of the Ag substrate on the aqueous solution of protonated PER at $\mathrm{pH} 2$. We prepared a $3 \times 10^{-4} \mathrm{M}$ aqueous solution of PER by mixing a suitable amount of a concentrated methanol solution of PER in water acidified with a mixture of $\mathrm{HCl}$ and $\mathrm{H}_{2} \mathrm{SO}_{4}$ in a 1:9 molar ratio. This expedient allows for limiting the quantity of $\mathrm{Cl}^{-}$in the solution. Indeed, we verified that excessive amounts of $\mathrm{Cl}^{-}$lead to the preferential formation of $\mathrm{Ag}-\mathrm{Cl}$ surface bonds. The $\mathrm{pH}$ was checked before adding PER. The SERS spectrum was recorded with $458 \mathrm{~nm}$ excitation wavelength through a $50 \times$ microscope objective $(\mathrm{NA}=0.75)$ at the solid-liquid interface formed by the solution droplet on the substrate, with $20 \mathrm{~s}$ exposure time ( 2 averages) and a laser power at the sample of $10 \mathrm{~mW}$, on a spot area of $1 \mu \mathrm{m}$ in diameter. 
(a)

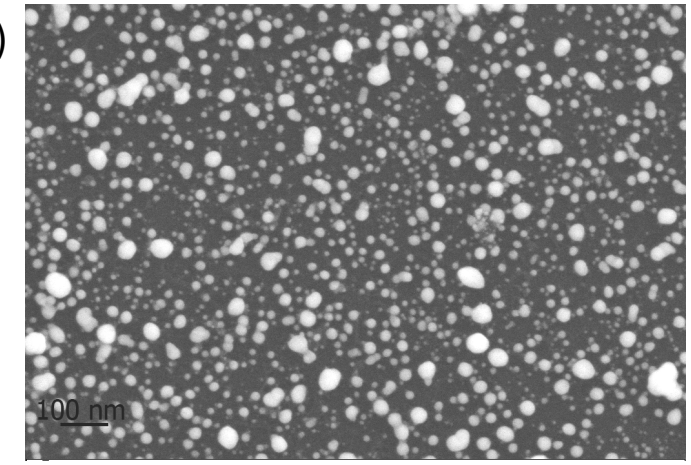

(b)

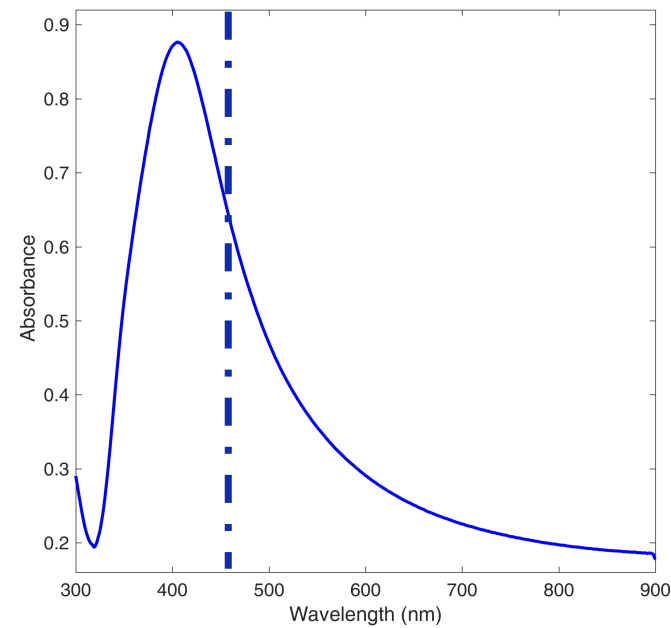

Figure 9. (a) surface nanostructure of an Ag substrate prepared by ps-PLAL at $f=1.5 \mathrm{~J} \cdot \mathrm{cm}^{-2}$ on a (100)Si support; (b) UV-vis spectrum taken on an analogous film sprayed on a glass support; the position of the exciting laser radiation is reported.

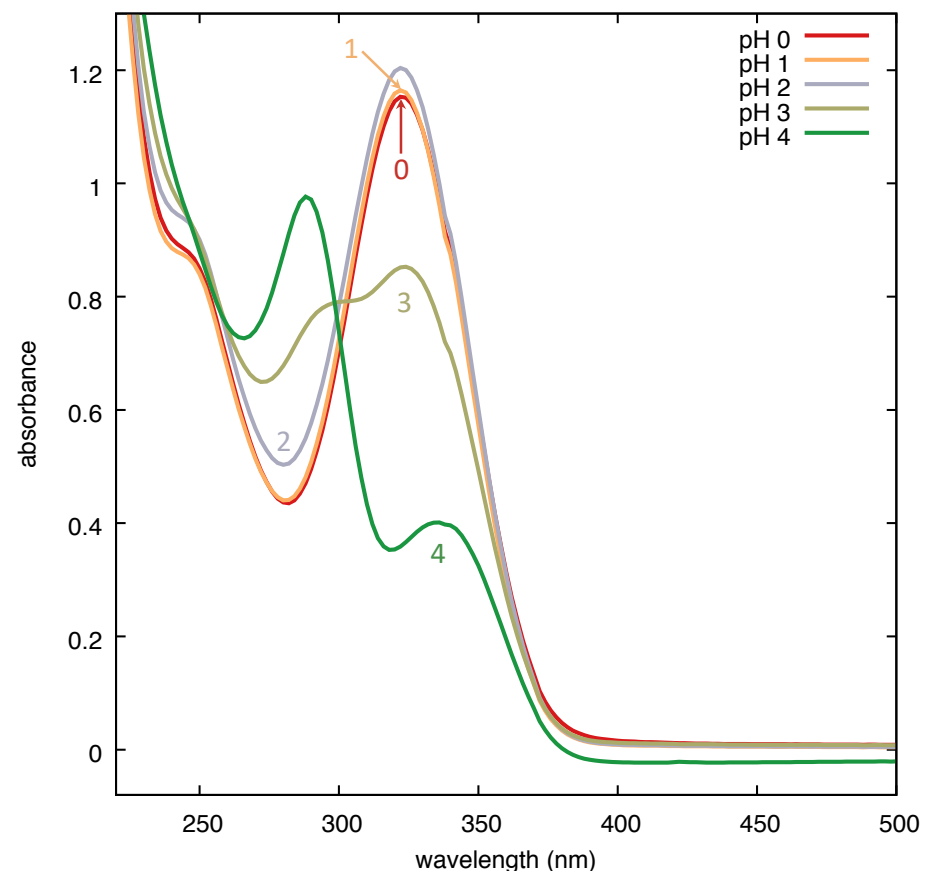

Figure 10. UV-Vis absorption spectra of aqueous solutions of Perampanel (PER) $\left(5 \times 10^{-5} \mathrm{M}\right)$ prepared at various $\mathrm{pH}$ ( $1 \mathrm{~cm}$ pathlength quartz cuvette, Jasco V-570 spectrometer). The label on each curve corresponds to the $\mathrm{pH}$ value. 
In Figure 11, the SERS spectrum is compared with the Raman spectrum of solid PER, as received (laser excitation $785 \mathrm{~nm}$ ). We observe that our early attempts to take SERS from neutral PER solutions failed. Remarkably, many of the major Raman features of solid PER appear in the SERS spectrum of an acidic PER solution (Figure 11). In particular, the following SERS lines of PER can be observed with a good signal to noise ratio: 666, 830, 877, 1000, 1018, 1158 (broad), 1225, 1279, 1394, 1447, 1483, 1514, $1599,2231 \mathrm{~cm}^{-1}$. Most of these lines match with those recently measured for protonated PER on Au substrates produced by PLA [24], namely 670 (666, this work), 875 (877), 1000 (1000), $1135(1158) \mathrm{cm}^{-1}$.

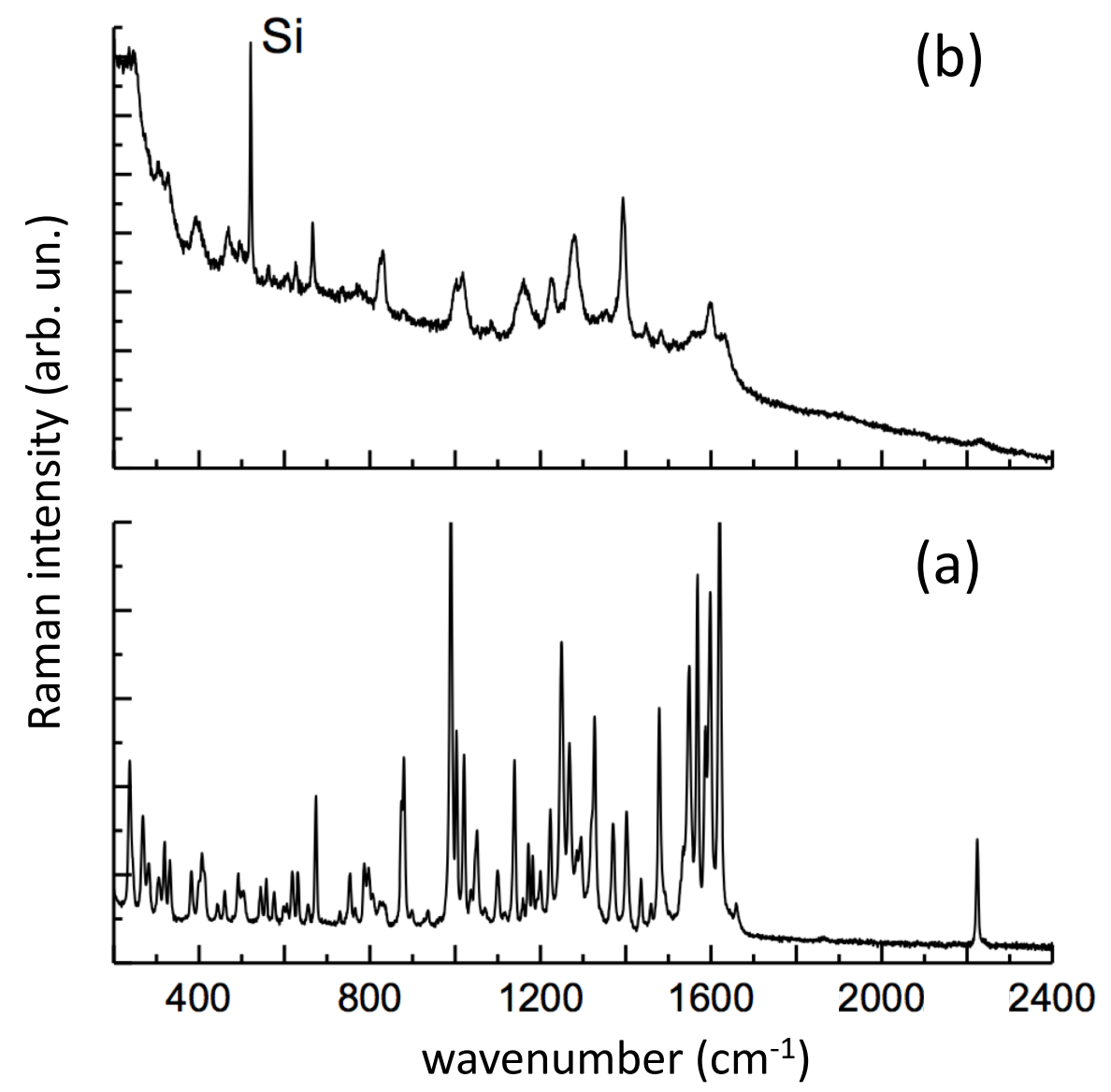

Figure 11. (a) Raman spectrum of solid Perampanel (PER) as received (785 nm excitation); (b) SERS spectrum of $3 \times 10^{-4} \mathrm{M}$ PER aqueous solution prepared at $\mathrm{pH} 2$ with a mixture of $\mathrm{HCl}: \mathrm{H}_{2} \mathrm{SO}_{4}$ in a 1:9 molar ratio. The SERS spectrum (458 nm excitation) was recorded at the solid-liquid interface of an $\mathrm{Ag}$ substrate prepared by ps-PLAL at $f=1.5 \mathrm{~J} \cdot \mathrm{cm}^{-2}$ on a (100)Si support.

Based on Density Functional Theory calculations (DFT B3LYP/6-31G(d,p)) [24], the principal Raman lines of PER can be assigned as in Table 2. The additional SERS lines of PER which can be detected with the Ag substrate produced by PLAL in this work are the following: 830, 1018, 1225, 1279, 1394, 1447, 1483, 1514, 1599, $2231 \mathrm{~cm}^{-1}$ (see Table 2 for details). Among these SERS signals, those assigned to a collective in-plane $\mathrm{C}-\mathrm{H}$ bending, coupled with collective ring deformations, can be considered as a fingerprint of PER. These SERS signals find their correspondence in the Raman spectrum of the solid $\left(1436,1478,1549,1569,1598,1619 \mathrm{~cm}^{-1}\right)$. The changes in relative intensity and the slight wavenumber shifts can be explained by the fact that SERS features belong to the protonated PER, whereas the Raman spectrum was recorded on the neutral species. 
Table 2. Assignment of the markers of Perampanel (PER) discussed in this work (after [24]).

\begin{tabular}{|c|c|}
\hline Wavenumber $\left(\mathrm{cm}^{-1}\right)$ & Description \\
\hline 666 & collective in-plane $\mathrm{C}-\mathrm{H}$ bending \\
\hline 830 & out-of-plane $\mathrm{C}-\mathrm{H}$ bending \\
\hline 877 & collective out-of-plane $\mathrm{C}-\mathrm{H}$ bending of the three outer rings of PER \\
\hline 1000 & trigonal ring deformation of the three outer rings of PER \\
\hline 1018 & ring deformation \\
\hline 1158 & collective $\mathrm{C}-\mathrm{H}$ in-plane bending of the ring carrying the $\mathrm{C} \equiv \mathrm{N}$ group \\
\hline 1225 & in-plane $\mathrm{C}-\mathrm{H}$ bending, ring deformation \\
\hline 1279 & in-plane $\mathrm{C}-\mathrm{H}$ bending, ring deformation of heterocycles \\
\hline 1394 & in-plane $\mathrm{C}-\mathrm{H}$ bending, central ring deformation \\
\hline 1447 & in-plane $\mathrm{C}-\mathrm{H}$ bending, pyridine ring deformation \\
\hline 1483 & collective in-plane $\mathrm{C}-\mathrm{H}$ bending coupled with collectve ring deformations \\
\hline 1514 & ring deformation of the only phenyl group of PER, in-plane $\mathrm{C}-\mathrm{H}$ bending \\
\hline 1599 & ring stretching \\
\hline 2231 & $\mathrm{C} \equiv \mathrm{N}$ stretching \\
\hline
\end{tabular}

Notably, the weak $C=O$ stretching peak of solid PER $\left(1658 \mathrm{~cm}^{-1}\right)$ disappears in the SERS spectrum of protonated PER. We deduce that the protonation of PER most likely occurs on the carbonyl.

\section{Conclusions}

We exploited the production by laser ablation of noble metal substrates engineered at the nanometer level with specific optical properties to perform SERS of selected AEDs toward a clinical application of the technique. An optimization of the substrate performance and the use of portable, possibly miniaturized Raman spectrometers are the next steps to address a point-of-care perspective.

Author Contributions: Conceptualization, M.T., F.N., P.M.O., S.T., E.F., M.C., E.C. and U.d.G.; methodology, C.Z., A.L., E.F. and S.T.; investigation, A.B., N.S.V., M.S., A.L., C.Z. and S.T.; resources, M.C., E.C. and U.d.G.; data curation, C.Z., E.F. and S.T.; writing, P.M.O., M.T. and C.Z.; supervision, M.T., P.M.O., C.Z., A.L., E.F. and F.N.

Funding: This research received no external funding.

Conflicts of Interest: The authors declare no conflict of interest.

\section{Abbreviations}

The following abbreviations are used in this manuscript:

$\begin{array}{ll}\text { AEDs } & \text { Antiepileptic Drugs } \\ \text { CBZ } & \text { Carbamazepine } \\ \text { FWHM } & \text { Full Width at Half Maximum } \\ \text { HPLC } & \text { High-Performance Liquid Chromatography } \\ N_{L P} & \text { number of laser pulses } \\ \text { NPs } & \text { nanoparticles } \\ \text { NTI } & \text { narrow therapeutic index } \\ p_{g} & \text { ambient gas pressure } \\ \text { PER } & \text { Perampanel } \\ \text { PLA } & \text { Pulsed Laser Ablation } \\ \text { PLAL } & \text { Pulsed Laser Ablation in Liquid } \\ \text { PLD } & \text { Pulsed Laser Deposition } \\ \text { SEM } & \text { Scanning Electron Microscopy } \\ \text { SERS } & \text { Surface Enhanced Raman Spectroscopy } \\ \text { SPR } & \text { Surface Plasmon Resonance } \\ \text { STEM } & \text { Scanning Transmission Electron Microscopy } \\ \text { TDM } & \text { Therapeutic Drug Monitoring } \\ \text { TEM } & \text { Transmission Electron Microscopy }\end{array}$




\section{References}

1. Solís, D.M.; Taboada, J.M.; Obelleiro, F.; Liz-Marzán, L.M.; García de Abajo, F.J. Optimization of Nanoparticle-Based SERS Substrates through Large-Scale Realistic Simulations. ACS Photonics 2017, 4, 329-337. [CrossRef]

2. Agarwal, N.R.; Ossi, P.M.; Trusso, S. Driving electromagnetic field enhancements in tailored gold surface nanostructures: Optical properties and macroscale simulations. Appl. Surf. Sci. 2019, 466, 19-27. [CrossRef]

3. Halas, N.J.; Moskovits, M. Surface-enhanced Raman spectroscopy: Substrates and materials for research and applications. MRS Bull. 2013, 38, 607-611. [CrossRef]

4. Ossi, P.M.; Bailini, A. Cluster growth in an ablation plume propagating through a buffer gas. Appl. Phys. A 2008, 93, 645-650. [CrossRef]

5. Neri, F.; Ossi, P.M.; Trusso, S. Cluster Synthesis and Assembling in a laser-generated plasmas. Riv. Nuovo Cim. 2011, 34, 103-149.

6. Tommasini, M.; Zanchi, C.; Lucotti, A.; Fazio, E.; Santoro, M.; Spadaro, S.; Neri, F.; Trusso, S.; Ciusani, E.; de Grazia, U.; et al. Laser Synthesized Nanoparticles for Therapeutic Drug Monitoring. In Advances in the Application of Lasers in Materials Science; Ossi, P.M., Ed.; Springer Nature Switzerland: Berlin, Germany, 2018; Chapter 12. [CrossRef]

7. Bailini, A.; Ossi, P.M. Expansion of an ablation plume in a buffer gas and cluster growth. EPL 2007, 79, 35002. [CrossRef]

8. Acacia, N.; Barreca, F.; Barletta, E.; Spadaro, D.; Currò, G.; Neri, F Laser ablation synthesis of indium oxide nanoparticles in water. Appl. Surf. Sci. 2010, 256, 6918-6922. [CrossRef]

9. Schwenke, A.; Wagener, P.; Nolte, S.; Barcikowski, S. Influence of processing time on nanoparticle generation during picosecond-pulsed fundamental and second harmonic laser ablation of metals in tetrahydrofuran. Appl. Phys. A 2011, 104, 77-82. [CrossRef]

10. Miotello, R.K.A. Comments on explosive mechanisms of laser sputtering. Appl. Surf. Sci. 1996, 96, $205-215$. [CrossRef]

11. Pathak, K.; Povitsky, A. Plume dynamics and shielding characteristics of nanosecond scale multiple pulse in carbon ablation. J. Appl. Phys. 2008, 104, 113108. [CrossRef]

12. Zheng, Y.B.; Kiraly, B.; Weiss, P.S.; Huang, T.J. Molecular plasmonics for biology and nanomedicine. Nanomedicine 2012, 7, 751-770. [CrossRef]

13. Hidi, I.J.; Mühlig, A.; Jahn, M.; Liebold, F.; Cialla, D.; Weber, K.; Popp, J. LOC-SERS: Towards point-of-care diagnostic of methotrexate. Anal. Methods 2014, 6, 3943-3947. [CrossRef]

14. Jaworska, A.; Fornasaro, S.; Sergo, V.; Bonifacio, A. Potential of Surface Enhanced Raman Spectroscopy (SERS) in Therapeutic Drug Monitoring (TDM). A Critical Review. Biosensors 2016, 6, 47. [CrossRef]

15. Litti, L.; Amendola, V.; Toffoli, G.; Meneghetti, M. Detection of low-quantity anticancer drugs by surface-enhanced Raman scattering. Anal. Bioanal. Chem. 2016, 408, 2123-2131. [CrossRef]

16. Zanchi, C.; Lucotti, A.; Tommasini, M.; Trusso, S.; de Grazia, U.; Ciusani, E.; Ossi, P.M. Laser tailored nanoparticle arrays to detect molecules at dilute concentration. Appl. Surf. Sci. 2017, 396, 1866-1874. [CrossRef]

17. Litti, L.; Ramundo, A.; Biscaglia, F.; Toffoli, G.; Gobbo, M.; Meneghetti, M. A surface enhanced Raman scattering based colloid nanosensor for developing therapeutic drug monitoring. J. Colloid Interface Sci. 2019, 533, 621-626. [CrossRef]

18. McLaughlin, C.; MacMillan, D.; McCardle, C.; Smith, W.E. Quantitative Analysis of Mitoxantrone by Surface-Enhanced Resonance Raman Scattering. Anal. Chem. 2002, 74, 3160-3167. [CrossRef]

19. Kuttner, C. Plasmonics in Sensing: From Colorimetry to SERS Analytics. In Plasmonics; Gric, T., Ed.; IntechOpen: Rijeka, Croatia, 2018; Chapter 9.

20. Muniz-Miranda, M.; Sbrana, G. Quantitative Determination of the Surface Concentration of Phenazine Adsorbed on Silver Colloidal Particles and Relationship with the SERS Enhancement Factor. J. Phys. Chem. B 1999, 103, 10639-10643. [CrossRef]

21. Yamamoto, Y.S.; Itoh, T. Why and how do the shapes of surface-enhanced Raman scattering spectra change? Recent progress from mechanistic studies. J. Raman Spectrosc. 2016, 47, 78-88. [CrossRef] 
22. Santoro, M.; Fazio, E.; Trusso, S.; Tommasini, M.; Lucotti, A.; Saija, R.; Casazza, M.; Neri, F.; Ossi, P.M. SERS sensing of perampanel with nanostructured arrays of gold particles produced by pulsed laser ablation in water. Med Devices Sens. 2018, 1, e10003. [CrossRef]

23. Morton, S.M.; Silverstein, D.W.; Jensen, L. Theoretical Studies of Plasmonics using Electronic Structure Methods. Chem. Rev. 2011, 111, 3962-3994. [CrossRef] [PubMed]

24. Zanchi, C.; Lucotti, A.; Tommasini, M.; Pistaffa, M.; Giuliani, L.; Trusso, S.; Ossi, P.M. Pulsed laser deposition of gold thin films with long-range spatial uniform SERS activity. Appl. Phys. A 2019, 125, 311. [CrossRef]

(C) 2019 by the authors. Licensee MDPI, Basel, Switzerland. This article is an open access article distributed under the terms and conditions of the Creative Commons Attribution (CC BY) license (http:/ / creativecommons.org/licenses/by/4.0/). 